\title{
Review of: "Production of minor ginsenosides by combining Stereum hirsutum and cellulase"
}

Yujun Liu ${ }^{1}$

1 Beijing Forestry University

Potential competing interests: The author(s) declared that no potential competing interests exist. 
Yang et al studied the production of $\mathrm{CK}$ from ginseng extracts by fermentation, and screened the optimum condition for SSF. This work firstly used S. hirsutum, a producer of $\beta$-glucosidase, to produce MGs from GE through SSF, which is innovative and meaningful for the production of MGs. However, there still exist numerous problems that should be addressed before publication.

Major concerns:

1. The use of abbreviation should be standardized. Abbreviations and full names should appear simultaneously in both the abstract and the body when it first appeared. After that, please use abbreviations. Please check these problems throughout the whole manuscript.

2. The HPLC profiles of various CE (including standards) should be provided.

3. The introduction of medicinal mushroom Stereum hirsutum should be added.

4. Results of Subsection 2.4 were exhibited in 3.4, an explanation should be added in 2.4 to connect the two subsections.

Sub-subsection 5.3.1 section said "Among them, four strains possessed the activity of transforming GE into CK as shown by HPLC analysis", but Fig. 2 only contained one strain, the remaining three strains should be added.

6. The study only focused on the content of MGs and CK, whether the bioactive abilities also enhanced or not is unclear. Besides, the yield content was improved by combining JE0512 with cellulase, whether or not the bioactive abilities also enhanced after combining JE0512 with cellulase is unclear. This problem should be addressed to enrich this article.

Minor concerns:

1. The typeface of primers of fungal $18 \mathrm{~S}$ ribosomal DNA ( $\mathrm{rDNA}$ ) should be normalized in Subsection 2.3.

2. Fig. 4 should be longer and Fig. 5 was two crowded. Please adjust them.

3. The letter "P" of $\mathrm{P}<0.05$ should be written in italics, please check this problem throughout the whole manuscript. 
